\title{
Keyhole Approach for Microscopic Excision of Third Ventricular Colloid Cyst: Our Experience
}

\author{
Sharad Samson Rajamani, Neeraj Salhotra*, Samit Biniwale, Bashar Al Tunbi, \\ Fahad Al Kheder, Faraz Qayyum Khan \\ Department of Neurosurgery, Khoula Hospital Muscat Oman \\ *Corresponding author: neersal@hotmail.com
}

Received November 10, 2019; Revised December 12, 2019; Accepted December 18, 2019

\begin{abstract}
Endoscopic surgery has truly revolutionized the surgical management of colloid cysts. Their central and deep location within the third ventricle has historically demanded a great degree of surgical skill and demand on the patient. Until the last two decades the treatment options for patients with colloid cysts included a traditional craniotomy (opening the skull for removal of the cyst), stereotactic cyst aspiration, or placement of a shunt for hydrocephalus.
\end{abstract}

Keywords: colloid cyst, key hole approach

Cite This Article: Sharad Samson Rajamani, Neeraj Salhotra, Samit Biniwale, Bashar Al Tunbi, Fahad Al Kheder, and Faraz Qayyum Khan, "Keyhole Approach for Microscopic Excision of Third Ventricular Colloid Cyst: Our Experience.” American Journal of Medical Case Reports, vol. 8, no. 2 (2020): 44-48. doi: 10.12691/ajmcr-8-2-2.

\section{Introduction}

Colloid cysts are benign tumors that most commonly appear in the third ventricle and account for $0.5-2.0 \%$ of all central nervous system tumors. They are composed of an outer fibrous layer and an inner epithelium of ciliated or mucin-producing cells. In patients presenting with symptoms the mortality is estimated to be $3.1-12 \%$. Overall mortality rate, regardless of presenting symptoms, is reported to be $1.2 \%(6,8)$. Nearly $60 \%$ of these mucinous cysts are found incidentally during routine work-up for other neurological diseases and are asymptomatic. In symptomatic cases, obstructive hydrocephalus may occur due to proximity with the foramen of Monro. Additional symptoms include coma, headaches, nausea, and vomiting. Patient age, headache as a primary symptom, and hydrocephalus are significantly associated with symptomatic colloid cysts. More than half of the patients presenting with symptoms will need surgical intervention. Surgical treatment is indicated for cysts that are symptomatic, large, or associated with hydrocephalus. Close observation is considered for small asymptomatic lesions .We present here our experience of microsurgical excision of colloid cyst via a minicraniotomy.

\section{Material and Methods}

We herewith present 10 patients operated by single surgeon via a minicraniotomy. All these patients presented with headache except one who came as deteriorated GCS which required intubation and emergency external ventricular drain insertion and once patient improved underwent definitive surgery. All patients underwent CT and MRI which revealed third ventricular colloid cyst. After explaining all possible risks and benefits patient underwent navigation assisted minicraniotomy of $2 \mathrm{~cm}$ diameter size via a trans sulcal approach through midfrontal gyrus with microsurgical excision of the cyst.

\section{Results}

A total of 10 patients were operated via this approach as outlined in table. Out of these 10 patients, 9 had been males and 1 being female. 9 patients had been having only headache and 1 presented with deterioration of sensorium . Outcome was excellent.

Table 1. Details of the patients operated

\begin{tabular}{|c|c|c|c|c|c|}
\hline S.No. & IP no. & Age/sex & Diagnosis & Approach & Outcome \\
\hline 1 & 875646 & $16 y r M$ & Colloid cyst & Minicraniotomy microsurgical excision & Good \\
\hline 2 & 348166 & 35yrM & Colloid cyst & Minicraniotomy microsurgical excision & Good \\
\hline 3 & 113404 & 78yr M & Colloid cyst & Minicraniotomy Microsurgical excision & Good \\
\hline 4 & 307411 & 43 yr. M & Colloid cyst & Minicraniotomy microsurgical excision & Good \\
\hline 5 & 558338 & 34yr M & Colloid cyst & Minicraniotomy microsurgical excision & Good \\
\hline 6 & 854534 & 47 yr M & Colloid cyst & Minicraniotomy microsurgical excision & Good \\
\hline 7 & 913958 & $37 \mathrm{yr} \mathrm{M}$ & Colloid cyst & Minicraniotomy microsurgical excision & Good \\
\hline 8 & 929874 & $32 \mathrm{yr} M$ & Colloid cyst & Minicraniotomy microsurgical excision & Good \\
\hline 9 & 938511 & 33 yr F & Colloid cyst & Minicraniotomy microsurgical excision & Good \\
\hline 10 & 1011219 & 35 yr M & Colloid cyst & Minicraniotomy microsurgical excision & Good \\
\hline
\end{tabular}



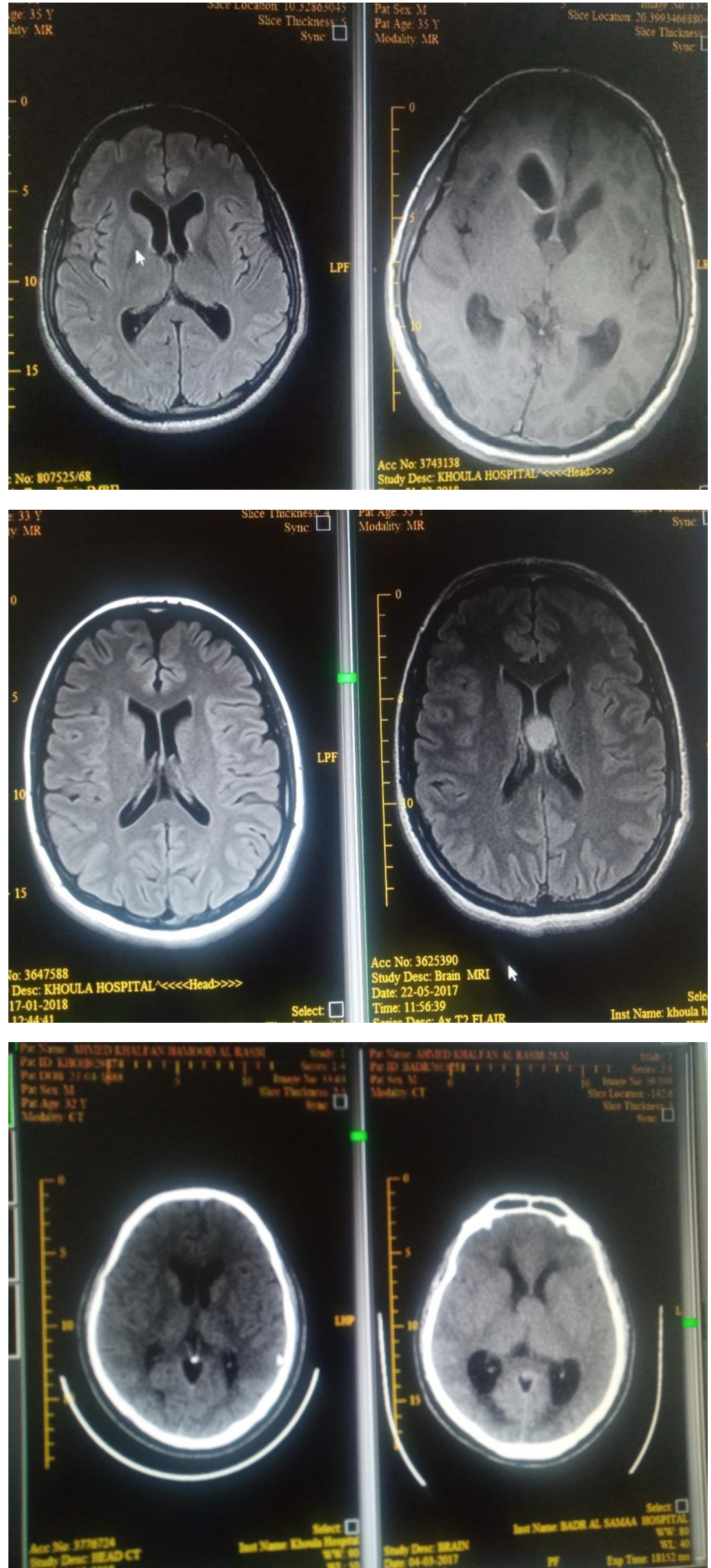

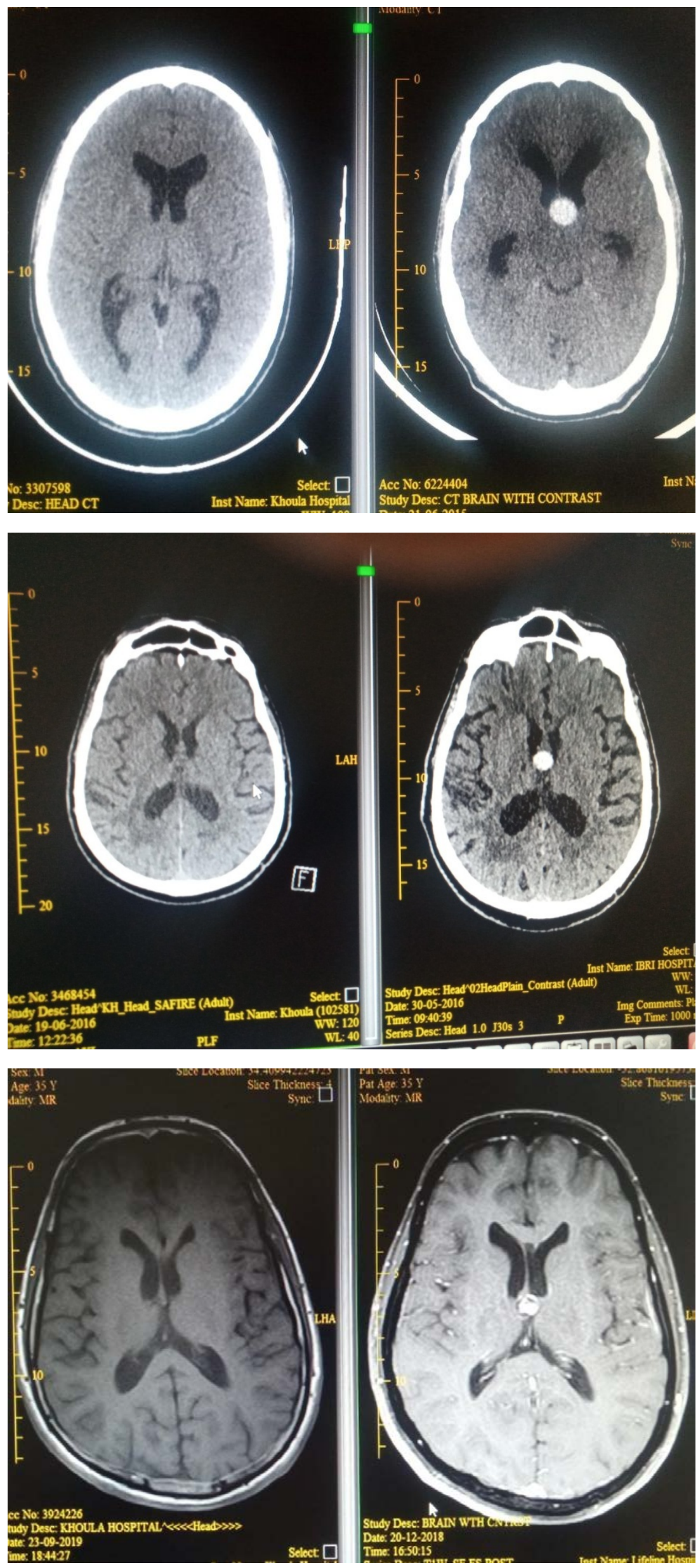

Figure 1. Pre and post op images of our patients with colloid cyst 

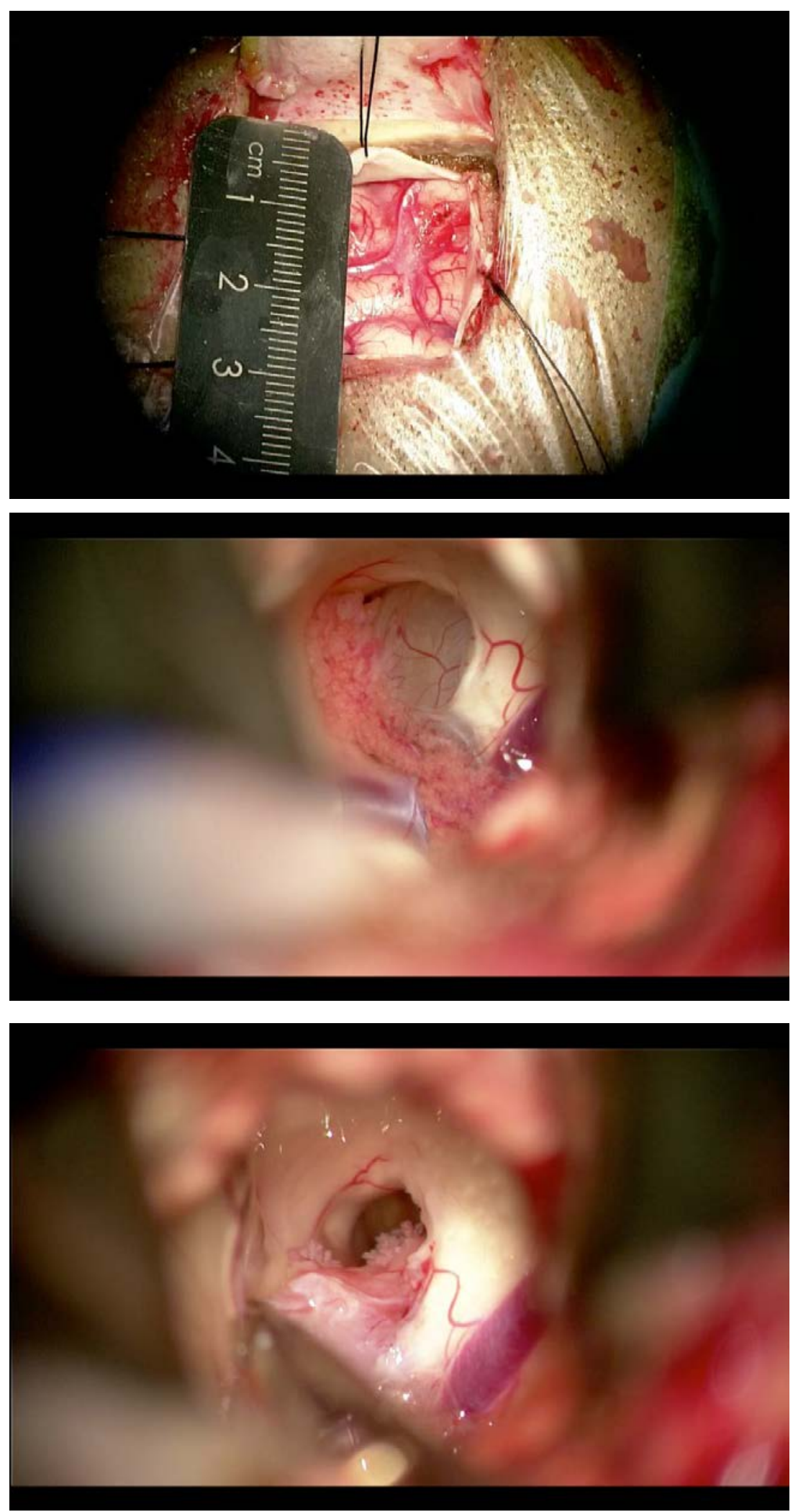

Figure 2. Intraoperative pictures of minicraniotomy, pre and post op excision of colloid cyst

\section{Discussion}

Haider G in 2017 studied many cases of colloid cysts and put down their guidelines for choosing patients for endoscopic removal or microscopic removal [1]. Shaikh AB in 2014 studied 1278 patients of colloid cyst and did a met analysis on both types of approach as endoscopic versus microscopic [2]. Spanchi PA in 2017 further studied their group of patients undergoing microsurgical and endoscopic approach es [3]. Cohen Gadol in 2013 described minitubular approach for colloid cyst excision [4]. Ailan AM in 2017 further described tubular endoscopic approach for colloid cyst excision [5]. Bergsnider M on 2007 gave his series of colloid cyst excision by using dual port endoscope [6]. 


\section{Conclusion}

Endoscopic excision of colloid cysts is currently well established as a minimally invasive and highly effective technique that is associated with less morbidity in comparison to microsurgical resection however minicraniotomy with microsurgical excision still holds a role with comparative results.

\section{References}

[1] Choosing between endoscopic or microscopic removal of third ventricle colloid cysts. Review article Haider G, et al. J Pak Med Assoc. 2017.
[2] Endoscopic versus microsurgical resection of colloid cysts: a systematic review and meta-analysis of 1,278 patients. Review article Sheikh AB, et al. World Neurosurg. 2014.

[3] Transcortical Removal of Third Ventricular Colloid Cysts: Comparison of Conventional, Guided Microsurgical and Endoscopic Approaches and Review of the Literature. Review article Sabanci PA, et al. Turk Neurosurg. 2017. Sabanci PA, Aras Y, Ali A, Unal TC, Dolen D, Sencer S, Izgi N, Unal F, Barlas O.

[4] Minitubular transcortical microsurgical approach for gross total resection of third ventricular colloid cysts: technique and assessment. Cohen-Gadol AA. World Neurosurg. 2013. CohenGadol AA.

[5] Endoscopic transtubular resection of a colloid cyst. Ajlan AM, et al. Neurosciences (Riyadh). 2014. Authors Ajlan AM, Kalani MA, Harsh GR.

[6] Complete microsurgical resection of colloid cysts with a dual-port endoscopic technique. Bergsneider M. Neurosurgery. 2007. Authors Bergsneider M.

(C) The Author(s) 2020. This article is an open access article distributed under the terms and conditions of the Creative Commons Attribution (CC BY) license (http://creativecommons.org/licenses/by/4.0/). 